\title{
An Evaluation of the Sieving Device YASD for 1024-bit Integers * (Extended Abstract)
}

\author{
Naoyuki Hirota ${ }^{1}$, Tetsuya Izu$^{2}$, Noboru Kunihiro ${ }^{1}$ and Kazuo Ohta ${ }^{1}$ \\ 1 The University of Electro-Communications, \\ 1-5-1, Chofugaoka, Chofu, 182-8585, Japan

\begin{abstract}
In 2004, Geiselmann and Steinwandt proposed a hardware design "YASD" for the sieving step in the number field sieve (NFS) method of integer factorization. This design is very attractive since its regular structure is suitable for implementation, however, its performance evaluation for 1024-bit integers was not studied. This paper attempts to evaluate the performance of YASD for 1024-bit integers based on the same assumptions of the orginal YASD. In our estimation, YASD for 1024-bit integers requires at least 16 years with cost $10 \mathrm{M}$ dollars.
\end{abstract}

\section{Introduction}

The RSA public-key cryptosystem is widely used as a de fact standard. It is strongly believed that breaking RSA is as hard as factoring large, say 1024bit or 2048-bit, integers. Thus the number field sieve (NFS) method, the best factoring algorithm, is an essential threat for the RSA cryptotsytem. Recently, NFS succeeded factoring a 633-bit integer by software implementation [RSA200]. Based on this fact, it is considered that factoring 1024-bit integers is infeasible in at least 10 years. However, this expectation does not take into account recent research results on dedicated factoring devices of NFS orginated from Berestein [Ber01]. NFS consists of 4 steps: the polynomial selection step, the relation finding (or sieving) step, the linear algebra step, and the fical step. Since the sieving step is the most time-cunsuming step, a lot of reserches have been focused on this step.

In 2003, Adi Shamir and Eran Tromer proposed an ASIC-based hardware design "TWIRL" for the sieving step [ST03]. According to their estimation, TWIRL could sieve within 90 days with cost 5000 dollars for a 768-bit integer. More surprisingly, TWIRL could factor even a 1024-bit integer within one year

* A part of this research is supported by a contract research with the National Institute of Information and Communications Technology (NICT), Japan 
with cost $10 \mathrm{M}$ dollars (with $1 \mathrm{GHz}$ frequency). Since TWIRL uses various kinds of cuircuit (irregular structure), it requires an LSI as large as a full wafer with $300 \mathrm{~mm}$ diameter.

On the other hand, in 2004, Willi Geiselmann and Rainer Steinwandt proposed another ASIC-based design "YASD" [GT04]. YASD is very attractive because of its small chip size and regular structure. According to the authors' estimation, this device requires about 300 days with cost 5000 dollars for a 768-bit integer (with $500 \mathrm{MHz}$ frequency). However, no estimation for 1024-bit integers have been provided up to the moment.

\section{Contribution of This Paper}

This paper focuses on YASD. In this paper, we evaluate the performance of YASD for 1024-bit integers (YASD1024) based on the same assumptions as those of TWIRL and YASD. Especially, we assumed that the process rule is $0.13 \mu \mathrm{m}$, the diameter of a full wafer is $300 \mathrm{~mm}$, the manufacturing cost of a wafer is 5000 dollars. As a result of our estimation, YASD1024 requires at least 16 years with cost $10 \mathrm{M}$ dollars (with $500 \mathrm{MHz}$ frequency). In the optimized case (in the sense of Area-Time product), YASD1024 requires an LSI as large as 411.17 $\mathrm{cm}^{2}$ with $k=28$ and $m=10$ (these variables will be defined later). Since we do not consider wiring problem in detail in our estimation, YASD1024 reuires much more time or/and cost even if it is manufactured and proceeded. Thus our estimation may be little better than the best case analysis.

The rest of this paper is as follows: in section 2, we briefly review the number field sieve (NFS) method of factorization and the structure of YASD. Then we analyze and parameterize YASD in section 3 and 4 . In section 5, we optimize these parameters for 1024-bit integers.

\section{Preliminaries}

In this section, we briefly review the number field sieve (NFS) method and the sieving device YASD for later discussion. For details of YASD, see the original paper [GT04].

\subsection{Number Field Sieving Method}

The number field sieving (NFS) method of integer factorization is the best algorithm, which consists of (1) the polynomial selection step, (2) the relation finding (or sieving) step, (3) the linear algebra step, and (4) the final step. Since (2) and (3) are most time-consuming steps in NFS, dedicated devices have been studied and proposed for these steps. In 2001, Bernstein employed a sorting algorithm for LA step with standard ASIC architectures [Ber01]. Then Lenstra, Shamir, Tomlinson and Tromer enhanced the device by using a routing algorithm [LSTT02]. On the other hand, Geiselmann and Steinwandt applied these algorithms to the 
Table 1. Sieving parameter [LTSK+03]

\begin{tabular}{|c|c|c|}
\hline & 768 -bit & 1024 -bit \\
\hline$H_{a}$ & $1.7 \times 10^{13}$ & $5.5 \times 10^{14}$ \\
$H_{b}$ & $8.9 \times 10^{6}$ & $2.7 \times 10^{8}$ \\
$B_{\mathrm{r}}$ & $10^{8}$ & $3.5 \times 10^{9}$ \\
$B_{\mathrm{a}}$ & $10^{9}$ & $2.6 \times 10^{10}$ \\
\hline
\end{tabular}

sieving step, and proposed two designs DSH [GT03] and YASD [GT04]. Shamir and Tromer improved an optical sieving device TWINKLE [Sha99] into a novel ASIC-based device TWIRL [ST03]. Recently, Franke, Kleinjung, Parr, Pelzl, Priplata and Stahlke proposed a challenging device SHARK for the sieving step based on the lattice sieving [FKPP +05$]$.

Let $n$ be an integer to be factored. In the beginning of NFS, two univariate polynomials $f_{\mathrm{r}}(x), f_{\mathrm{a}}(x)$ and an integer $m$ such that $f_{\mathrm{r}}(m) \equiv f_{\mathrm{a}}(m) \equiv 0$ $(\bmod n)$ are selected in the polynomial selection step. Then these polynomials are converted to bivariate and homogeneous polynomials $F_{\mathrm{r}}(x, y), F_{\mathrm{a}}(x, y) \in$ $\mathbb{Z}[x, y]$. The purpose of the relation finding step is to find a large number of relations, namely coprime integer pairs $(a, b) \in\left[-H_{a}, H_{a}\right] \times\left[1, H_{b}\right] \subset \mathbb{Z} \times \mathbb{N}$ such that $F_{\mathrm{r}}(a, b)$ is $B_{\mathrm{r}}$-smooth and $F_{\mathrm{a}}(a, b)$ is $B_{\mathrm{a}}$-smooth. Here an integer $x$ is called $B$-smooth if $x$ is factored over the primes less than $B$. Procedures corresponding to $F_{\mathrm{r}}\left(F_{\mathrm{a}}\right)$ are sometimes called 'rational' ('algebraic'). Parameters $H_{a}, H_{b}$ determines the sieving region for factoring, and depend on the size of the target integer $n$. In this paper, we use the following values as in Table 1 [LTSK+03]. In practicve, the core sieving step picks up possible relations (called candidates), and the additional step checks whether it really is a relation by mini-factoring algorithms. After finding a set of relations, Gaussian elimination over a matrix generated from these relations is computed in the linear algebra step. Then, a factor of $n$ is output in the final step.

\subsection{YASD}

YASD (Yet Another Sieving Device) is a dedicated sieving device of NFS proposed by Geiselmann and Steinwandt in 2004 [GT04]. YASD consists of a mesh $\left(2^{m} \times 2^{m}\right)$ of processing nodes, where each node is connected to its horizontal and vertical neighbors. Each node conceptually has three parts: the Main part, the Mesh part, and the Memory part, and 2 buffers between the Main part and the Mesh part, and between the Mesh part and the Memory part. In YASD, the sieving process is achieved by sending packets from the Main part of certain nodes to the Memory part of certain nodes (target nodes). Each Mesh part of a node can hold a packet. Information of the target nodes are in the packets, and the packets are routed via the Mesh parts controled by the routing algorithm. Memory parts of a mesh can proceed $S=2^{k}$ sieving lines in one process, so each node is in charge of $Z=2^{k-2 m}$ consecutive sieving positions (integer pairs). 


\begin{tabular}{|c|c|c|c|c|c|}
\hline$\left(x_{t}, y_{t}\right)$ & $c_{x}, c_{y}$ & $\left\lceil\log _{\sqrt{2}} p\right\rceil$ & footprint & $z_{t}$ & flag \\
$(16)$ & $(2)$ & $(6)$ & $(26)$ & $(8)$ & $(1)$ \\
\hline
\end{tabular}

Fig. 1. Packet Structure

Table 2. Dispersive storing of the factor base

\begin{tabular}{|c|c||c|c|}
\hline size of $p$ & storing density & size of $p$ & storing density \\
\hline $2 \sim 2^{8}$ & $1 \times 1$ & $2^{14} \sim 2^{16}$ & $2^{4} \times 2^{4}$ \\
$2^{8} \sim 2^{10}$ & $2 \times 2$ & $2^{16} \sim 2^{18}$ & $2^{5} \times 2^{5}$ \\
$2^{10} \sim 2^{12}$ & $2^{2} \times 2^{2}$ & $2^{18} \sim 2^{20}$ & $2^{6} \times 2^{6}$ \\
$2^{12} \sim 2^{14}$ & $2^{3} \times 2^{3}$ & $2^{20} \sim B_{1}$ & $2^{8} \times 2^{8}$ \\
\hline
\end{tabular}

Main Part A main function of the Main part is to generate packets from the factor base (stored in the Main part) and to send packets to the Mesh Part. More precisely, for a prime $p$ in the factor base, the Main part outputs integers $r$ such that $f_{i}(r) \equiv 0(\bmod p)(i=1,2)$. These packets have a common property that if $(p, r)$ is a proper packet, then $(p, r+p)$ is also a proper packet. If $(p, r+p)$ is in the current sieving subinterval $S$, this part sends the packet which holds $(p, r+p)$ to the Mesh Part. Otherwise, the same process is performed for the next prime $p^{\prime}$.

Figure 1 shows a packet structure generated by the Main Part. Since nodes are arranged in a square, each node is addressed in $(x, y)$-coordinates. In, Figure $1,\left(x_{t}, y_{t}\right)$ is a coordinate values of its target node and $z_{t}$ is a sieving position in which the packet will be stored. $c_{x}, c_{y}$ are cross border flags when a torus topology is used in the mesh. A "footprint" is the factoring information which consists of the coordinates of the target node (16-bit) concatenated with its 10 lower bits of $p$ (more precisely, the lowest bit ' 1 ' is excluded since $p$ is prime). This information is used in postprocess called the "mini-factoring".

The coordinate values of the target node $\left(x_{t}, y_{t}\right)$ and target sieving position $z_{t}$ is computed in the Main part. If the target node is itself, namely the current node and the target node are same, the packet is directly sent to the input buffer which connect to the Main Part and the Memory Part. When a packet is sent from the input buffer to the Mesh Part, 25\% nodes are kept empty in order to avoid packet congestion,

The factor base is dispersively stored in $2^{m} \times 2^{m}$ nodes. At this time the factor bases about $p$ such that $p<Z\left(=2^{k-2 m}\right)$ are stored in all nodes because such factor bases are necessarily used in all nodes. Also, the factor bases about $p$ such that $Z<p<2^{k-2 m+2}$ are stored sharing with $2^{2}$ nodes, and those such that $2^{k-2 m+2}<p<2^{k-2 m+4}$ are stored sharing with $2^{4}$ nodes. At last, those such that $p>2^{k}$ are stored only once in the mesh (See Table 2). 
Memory Part The Memory part of each node has two 10-bit DRAM entries for each of $256 z$-values that the node has to be taken care of. One DRAM is for the algebraic side and the other is for the rational side. These memories are initialized with 0 and hold sums of $\left\lceil\log _{\sqrt{2}} p\right\rceil$-values. In addition, the Memory part stores footprints in the special DRAM. This DRAM is shared among two neighbornodes. Thus, when storing footprints, in addition to 26-bit for footprint itself, 8-bit of sieving position $z_{t}$, 1-bit algebraic/rational flag, and 1-bit to identify the node. Totally, one footprint requires 36-bit to be stored. As well as the Main part, the Memory part requires about two receiving buffer connecting the Memory part and the Mesh part. Differing from the Main part case, 41-bit is sufficient for this buffer because target nodes and cross border flags are no longer needless.

Mesh Part A main function of the Mesh part is to route packets to their target nodes. The routing is controlled by the clockwise transposition routing algorithm and a complete logic is located in the Mesh part. This part needs two 59-bit register to hold 'traveling packets' on the mesh. According to the analysis by Lenstra et al. [LSTT02], a clockwise transposition routing over an $L \times L$-mesh requires at most $2 L$ steps for a non-torus mesh and at most $2(L-1)$ steps for a torus mesh.

\section{Analysis of YASD}

In [GT04], Geiselmann and Steinwandt analyzed the performance of YASD for 768-bit integers (YASD768). According to their estimation, an LSI of YASD 768 requires as large as $4.9 \mathrm{~cm} \times 4.9 \mathrm{~cm}$ and the running time is about 600 days at $500 \mathrm{MHz}$ frequency. However, they refer only to above values of YASD without explanation. They don't mention about the reason of these values logically. So, in this section, we will analyze makeup of the circuit based on its operation and function, and verify their claim for 768-bit.

\subsection{Chip Size of YASD}

Table 3 shows the number of circuit element, DRAM and transistor, needed in each part. This numbers mentioned in [GT04].

In addition to above numbers, with a $0.13 \mu \mathrm{m}$ process, we have to take $0.3\left[\mu \mathrm{m}^{2}\right]$ for a DRAM bit and $2.8\left[\mu \mathrm{m}^{2}\right]$ per transistor into account [GT04]. Using these numbers, the size of chip is formulated as below.

$$
\text { Area }=(5100 \times 2.8+66500 \times 0.3) \times 256^{2}=22.4\left[\mathrm{~cm}^{2}\right]
$$

Therefore, we say that the claim of [GT04] is appropriate. And the difference of claimed number from calculated number by above formulation $1.6\left[\mathrm{~cm}^{2}\right]$ can be considered as Input/Output circuit proportional to the number of node. 
Table 3. The number of circuit element needed in each part

\begin{tabular}{|c|c|c|}
\hline & Transistor & DRAM \\
\hline Main Part & 2750 & 55000 \\
Memory Part & 1250 & 11500 \\
Mesh Part & 1100 & - \\
\hline per node & 5100 & 66500 \\
\hline
\end{tabular}

\section{$3.2 \quad$ Performance of the Device}

In reference [GT04], it is described that the sieving process for the range $S=$ $2^{24}$ that YASD can deal with at once takes 40000 clocks. If we analyze this number, we need to take routing time $T_{\text {rout }}$ for clockwise transposition routing and sending time $T_{\text {send }}$ at the Main Part into account. Sending time $T_{\text {send }}$ depends on the number of packet which is needed to sent to the Mesh Part.

Sending Time $\boldsymbol{T}_{\text {send }}$ To formulate this time $T_{\text {send }}$, we divide primes into three groups according to its size. At first, let $2^{m} \times 2^{m}$ be mesh size of YASD and $2^{k}$ be the size of sieving range which YASD can deal with at once. Next, in case of generating packets which is calculated from pairs $(p, r)$ such that $p<Z$, in average, $Z / p$ times calculation of $p$ is needed. Describing in section 2.2 , in case of primes such that $Z<p<2^{k}$, these pair $(p, r)$ is stored as describing Table 2 to share them among neighbor nodes. For example, primes such that $2^{k-2 m+2 i}<p<2^{k-2 m+2(i+1)}(i=0, \ldots, m-1)$ will take charge the sieving range of length $2^{k-2 m+2(i+1)}$, thus in average, $2^{k-2 m+2(i+1)} / p$ times calculation concerning this $p$ is needed. And such pairs are shared among $2(i+1)$ nodes. Therefore, the calculating time of all primes such that $Z<p<2^{k}$ is $\sum_{Z<p<2^{k}} \frac{Z}{p}$ per node. Concerning primes such that $2^{k}<p<B_{\text {a }}$ at most one calculation is needed. Consequently, concerning primes such that $p<2^{k}$, the necessary number of calculation for prime sending which is dealt with one node is $2 \sum_{p<2^{k}} \frac{Z}{p}$ (putting together algebraic and rational side). Likewise, concerning primes such that $p>2^{k}, \frac{\pi\left(B_{\mathrm{a}}\right)+\pi\left(B_{\mathrm{r}}\right)-2 \pi\left(2^{k}\right)}{2^{2 m}}$ times of calculation are needed.

Especially, the calculation of primes such that $p>2^{k}$ requires 2 steps. So, sending time $T_{\text {send }}$ is formulated as Eq. (1).

$$
T_{\text {send }}=2 \sum_{p<2^{k}} \frac{Z}{p}+\frac{\pi\left(B_{\mathrm{a}}\right)+\pi\left(B_{\mathrm{r}}\right)-2 \pi\left(2^{k}\right)}{2^{2 m-1}}
$$

By substituting $Z=256, m=8, k=24$ into Eq. (1), $T_{\text {send }}$ is $\simeq 2968$ clocks. This time is rather smaller than 40000 clocks. So, $T_{\text {send }}$ is negligible and routing time $T_{\text {rout }}$ described later is dominant. 
Routing Time $\boldsymbol{T}_{\text {rout }}$ At first, when we evaluate the routing time, we check up this time base on practical analysis that it takes at most $2 L$ steps for routing at $L \times L$-mesh. Next, in this mesh, the number of packet which is needed to send is expressed as follows.

$$
\sum_{Z<p<B_{\mathrm{a}}} \frac{2^{k}}{p}+\sum_{Z<p<B_{\mathrm{r}}} \frac{2^{k}}{p}, \text { where } p \text { is a prime }
$$

The values $B_{\mathrm{a}}, B_{\mathrm{r}}$ for 768 -bit are respectively $B_{\mathrm{a}}=10^{9}, B_{\mathrm{r}}=10^{8}$, so routing time $T_{\text {rout }}$ is $\simeq 4.226 \times 10^{7}$ from above equation. Then we use values $m=8, k=24$, which are derived from [GT04].

And we think of following assumption.

1. the mesh size is $2^{m} \times 2^{m}$.

2. Initially, the mesh is filled with $2^{m} \times 2^{m}$ packets.

3. We don't throw new packets in the mesh until the entire first packet are routed.

We apply to this model and suppose that one routing time is $2^{m}$ steps in average. In addition to above model, the YASD device applies to torus topology. According to our software simulations, if applied to torus topology, the YASD can route packets more than twice faster than so-called 'primitive' model. Furthermore, to avoid packet congestion on mesh, it is applied to more efficient releasing rule. In this way usually $\simeq 25 \%$ of the node are 'free' (see Figure 2 ) and nearly twice faster. Therefore, we add the coefficient $\alpha$ that derive from above speeding up method and let $\alpha$ be $\simeq \frac{1}{4}$. So, the routing time $T_{\text {rout }}$ becomes $\alpha \times 4.226 \times 10^{7} / 2^{8}=41269$ steps. This value is nearly 40000 clocks. So, we adopt 0.242 as the coefficient $\alpha$.

Consequently, the routing time $T_{\text {rout }}$ can be written as Eq. (3).

$$
T_{\text {rout }}=\frac{\sum_{Z<p<B_{\mathrm{a}}} \frac{2^{k}}{p}+\sum_{Z<p<B_{\mathrm{r}}} \frac{2^{k}}{p}}{2^{m}} \times \alpha \quad \text {, where } \alpha=0.242
$$

\section{Formulation of the Performance}

In this section, we estimate chip size and the performance time of YASD using 1024-bit parameter. The 1024-bit parameter is described at Table 1.

At first, we analyze the detail of the number of circuit element from 768-bit composition. Table 4 shows the result of this analysis for 1024-bit.

By using variables in Table 4, bit length of the packet $P A C$ is expressed as $P A C=k+L_{\log }+2 m+10+3$ (we use this variable $P A C$ later), where fourth term of right-hand member 10 is derived from footprint information and last term 3 is assigned to flag (see Table 1 ).

In addition, from reference [GT04,GT03], Table 5 shows the number of transistor needed for respective logic. 


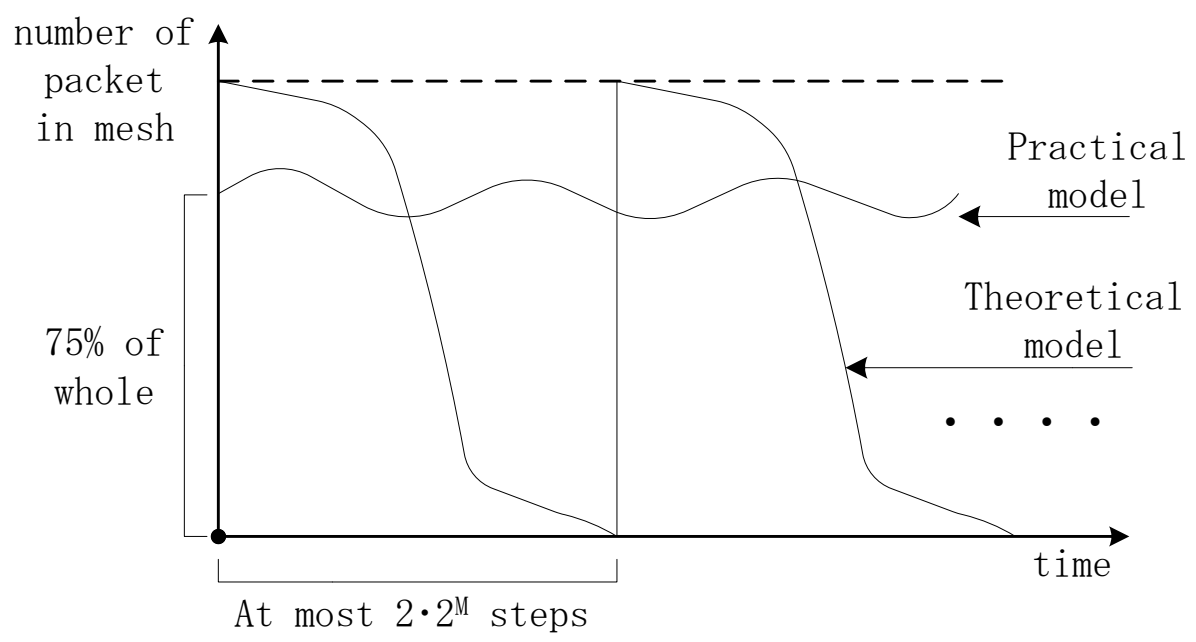

Fig. 2. Routing model

Table 4. Analysis Results

\begin{tabular}{|c|c|c|c|}
\hline \multicolumn{2}{|c|}{ Variables } & 768 -bit & 1024 -bit \\
\hline$L_{p}$ & bit length of maximal prime & 30 & 32 \\
$L_{\log }$ & bit length of $\left\lceil\log _{\sqrt{2}} p\right\rceil$ & 6 & 8 \\
$L_{\mathrm{mem}}$ & bit length of $\sum_{p}\left\lceil\log _{\sqrt{2}} p\right\rceil$ & 10 & 11 \\
$N$ & total number of factor bases & $7.97 \times 10^{7}$ & $1.32 \times 10^{9}$ \\
$F$ & total number of footprint & $0.496 \times 2^{k}$ & $0.818 \times 2^{k}$ \\
$L_{\mathrm{fb}}$ & average of bit length of factor base & 38 & 42 \\
\hline
\end{tabular}

\subsection{Analysis of the Number of Circuit Element}

Recall that the number of circuit element is described in Table 3. In this part, we describe more detail about the number of circuit element of each part and formulate respectively.

The Number of Transistor for the Main Part For 768-bit integers, the Main Part needs 2750 transistors. The purpose of the Main Part is to generate packets from factor bases $(p, r)$ and send to the Mesh Part. So, it needs $\max \left(L_{p}, k\right)$-bit adder for generating packets and buffers between Main-Mesh Part for sending. According to [GT04], two entry buffers are sufficient. And

${ }^{3}$ D-F/Fs make up resister.

${ }^{4}$ Latches make up buffer.

${ }^{5}$ we guessed this value from $H_{\text {add }}$ of reference [GT03], so more precise discussion is needed. 
Table 5. The number of transistor needed for respective logic

\begin{tabular}{|c|c|c|}
\hline variable & logic & the number of transistor \\
\hline$H_{\text {add }}$ & 1-bit adder & 40 \\
$H_{\text {dff }}$ & 1-bit D-F $/ \mathrm{F}^{3}$ & 8 \\
$H_{\text {latch }}$ & 1-bit Latch & 4 \\
$H_{\text {comp }}$ & 1-bit comparator & 20 \\
\hline
\end{tabular}

so forth, Main Part needs circuit to initialize information of factor bases. Then we estimate the number of this circuit to be a constant number. Totally, we can formulate the number of transistors at Main Part as Eq. (4).

$$
T r_{\text {main }}=H_{\text {add }} \times \max \left(L_{p}, k\right)+2 \cdot P A C \times H_{\text {latch }}+1000
$$

About the constant number of right-hand member, we also need more discussion.

The Number of DRAM for the Main Part The DRAM of the Main Part is used for holding information of factor bases. The total number of factor bases $N$ depend on bit length of composite integer $n$. In YASD, each node holds this factor bases dispersively. Therefore, we estimate the number of DRAM as Eq. (5). At this equation, coefficient 1.1 means memory margin.

$$
D R A M_{\text {main }}=L_{\mathrm{fb}} \times \frac{N}{2^{2 m}} \times 1.1
$$

The number of Transistor for the Memory Part The function of the Memory Part is to extract $\left\lceil\log _{\sqrt{2}} p\right\rceil$ and $z_{t}$ from packet and add the value $\left\lceil\log _{\sqrt{2}} p\right\rceil$ to certain address represented as $z_{t}$, so it needs $L_{\text {mem-bit adder. And }}$ it also needs buffers between Mesh-Memory Part. In this case, target address $\left(x_{t}, y_{t}\right)$ and cross border flags $\left(c_{x}, c_{y}\right)$ are no longer needless because the packet has already reached to target node. Therefore, the bit length $P A C-2 m-2$ is sufficient for the buffer between Mesh-Memory Part and two entry buffers are sufficient as well as Main Part. Finally, we consider that the number of circuit to output sieving result from memories is constant. Consequently, we can formulate the number of transistor for Memory Part as Eq. (6).

$$
T r_{\text {mem }}=H_{\text {add }} \times L_{\text {mem }}+2 \cdot(P A C-2 m-2) \times H_{\text {latch }}+500
$$

The Number of DRAM for the Memory Part The Memory Part provides

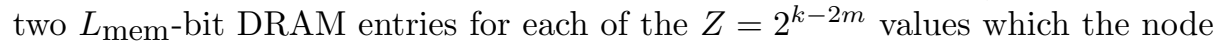
has to take care of. The 'two' means the algebraic and the rational side. And it needs the DRAM for footprint. According to [GT04], it is efficient to share the DRAM for footprint among two nodes. In this discussion, we only think 
the number of the DRAM per node. A footprint consist of target node address $\left(x_{t}, y_{t}\right)$ and the bits no. 1-10 of $p$. Since $p$ is prime, the least significant bit are always set. So actually footprint holds the least 10 bits of $\frac{p-1}{2}$. However, at the time of storing footprint, in addition to footprint itself, $Z=2^{k-2 m}$-bit of target sieving position, 1-bit of the algebraic/rational side flag, and 1-bit flag to identify the node are needed. Therefore, the total bit length for storing footprint is 38-bit. Although the average number of footprint stored in a node is about 127 by software calculation, the reference [GT04] says that it needs 325 entries for footprint among two nodes. So we take this into account and formulate as Eq. (7). The Coefficient 1.3 derives from $325 /(2 \cdot 127)$.

$$
D R A M_{\text {mem }}=2 \cdot Z \times L_{\text {mem }}+\frac{F}{2^{2 m}} \times(k+10+2) \times 1.3
$$

The Number of Transistor for the Mesh Part The function of the Mesh Part is compare/exchange operation. Exchanging logic consists of two $P A C$ bit resisters. Although it needs the circuit which take care of cross border flag $\left(c_{x}, c_{y}\right)$, we consider that its circuit is as small as negligible.

$$
T r_{\text {mesh }}=2 \cdot P A C \times H_{\text {dff }}+m \times H_{\text {comp }}
$$

Changes of $\boldsymbol{L}_{p}, \boldsymbol{L}_{\mathrm{mem}}, \boldsymbol{L}_{\mathrm{fb}}$ These variables are changed when we change factoring number $n$ from 768 -bit to 1024-bit. We discuss about these variables in this part.

$\left[L_{p}\right]$ For 1024-bit, the smoothness bound of algebraic side $B_{1}$ is $2.6 \times 10^{10}$. So, we can consider maximal prime as this value, so that $L_{p}$ is $\left\lceil\log _{2} \log _{\sqrt{2}}(2.6 \times\right.$ $\left.\left.10^{10}\right)\right\rceil=7$. Although 7-bit is sufficient for $L_{p}$, we have to take that $L_{p}$ holds sum of ceiling value into account. So we can say that 8 -bit is adequate for $L_{p}$.

[Lmem] We discuss the maximal value of sum of $\left\lceil\log _{2} \log _{\sqrt{2}} p\right\rceil$. This value is originally derives from $\simeq F(a, b)$. $F(a, b)$ is generated from $f(X)$ referred to [LTSK+03]. The order of the maximal value of $F(a, b)$ is about $10^{105}$. This value is given by substituting $a=A=5.5 \times 10^{14}, b=B=2.7 \times 10^{8}$. Therefore, $L_{\text {mem }}$ becomes $\left\lceil\log _{2} \log _{\sqrt{2}}\left(10^{105}\right)\right\rceil=10$. Since $L_{\text {mem }}$ also holds the sum of ceiling value, 11-bit is adequate for $L_{\mathrm{mem}}$.

$\left[L_{\mathrm{fb}}\right]$ The factor base information consist of the difference of consecutive primes $\frac{\Delta p}{2}$ and the root $r$. The majority of factor bases is so-called Hugish prime, which is $p>B_{\mathrm{r}}$ and so only has algebraic root ${ }^{6}$. So, about $\frac{\Delta p}{2}$, it is sufficient to make 2-bit longer as well as $L_{\log }$. And about algebraic root $r$, it is sufficient to make 2-bit longer as well as $L_{p}$. Summing up these, the bit length of factor base $L_{\mathrm{fb}}$ can be considered as 42-bit.

\footnotetext{
${ }^{6}$ The data structure of Hugish prime is as $\frac{\Delta p}{2}$ algebraic root $\mid$ flag.
} 


\subsection{Area Formula}

From above discussions, we can formulate the area of general YASD (for $N$-bit integers) as follows.

$$
\begin{aligned}
& \operatorname{Area}^{(N)}(k, m)=\left\{2.8 \times\left(68 m+72 k+32 L_{\log }+\right.\right.\left.40 L_{m e m}+1916\right) \\
&\left.+0.3 \times\left(1.1 L_{f b} \frac{n}{2^{2 m}}+2^{k-2 m+1} L_{m e m}+1.3(k+12) \frac{F}{2^{2 m}}\right)\right\} \times 2^{2 m} \\
&+2000 \times 2^{2 m} \quad\left(\mu \mathrm{m}^{2}\right)
\end{aligned}
$$

Here, $2000 \times 2^{2 m}$ corresponds to Input/Output circuit proportional to the number of node (see section 3.1).

\section{Analysis of YASD for 1024-bit Integers}

In this section, we formalize the circuit area and processing time of YASD for 1024-bit integers (YASD1024). Then we optimize the parameters $k, m$ in the sense of Area-Time product.

\subsection{Circuit Area}

By substituing parameters in Table 4 into the equation (9), we obtain the following formula of circuit area for YASD1024:

$$
\begin{aligned}
\text { Area }^{(1024)}(k, m)=(162.4 m+89.6 k+12959.2) \times 2^{2 m} & \\
& +(0.321 k+9.246) \times 2^{k}+1.83 \times 10^{10} \quad\left(\mu \mathrm{m}^{2}\right)
\end{aligned}
$$

\subsection{Processing Time}

According to the equation (3), the total number of packet being sent is as follows.

$$
\sum_{p>Z}^{2.6 \times 10^{10}} \frac{2^{k}}{p}+\sum_{p>Z}^{3.5 \times 10^{9}} \frac{2^{k}}{p}=(6.27-2 \log \log Z) \times 2^{k}
$$

Therefore, the routing time $T_{\text {rout }}(k, m)$ is given as Eq.12.

$$
T_{\text {rout }}(k, m)=\left(6.24-2 \log \log 2^{k-2 m}\right) \times 2^{k-m} \times 0.24
$$

In addition, since the sending time $T_{\text {send }}$ is obtained from Eq. (1), the number of steps needed for one sieving process can be obtained.

In NFS, relations are collected from a region $-H_{a} \leq a \leq H_{a}, 1 \leq b \leq H_{b}$. However, there is condition that the pair $(a, b)$ must be coprime. So in this region, if $a$ and $b$ are both even number, it is no necessity to sieve for such $a$ and $b$. By this means, $25 \%$ of the whole sieving region can be excluded.

And in order to compare to the TWIRL, we assume the condition as below. 
Table 6. Area and time values

\begin{tabular}{|c|c|c|c|c|c|c|c|c|c|c|}
\hline & & $m=5$ & 6 & 7 & 8 & 9 & 10 & 11 & 12 & 13 \\
\hline \multirow{3}{*}{$k=22$} & Area $\left(\mathrm{cm}^{2}\right)$ & 184 & 184 & 186 & 194 & 227 & 357 & & & \\
\hline & Time (year) & 365 & 216 & 128 & 77 & 48 & 32 & & & \\
\hline & AT product & 67044 & 39729 & 23863 & 14988 & 10832 & 11355 & & & \\
\hline \multirow{3}{*}{24} & Area $\left(\mathrm{cm}^{2}\right)$ & 186 & 187 & 189 & 197 & 229 & 361 & 895 & & \\
\hline & Time & 309 & 182 & 108 & 64 & 39 & 24 & 16 & - & \\
\hline & AT product & 57392 & 34006 & 20314 & 12590 & 8842 & 8634 & 14215 & & \\
\hline \multirow{3}{*}{26} & Area $\left(\mathrm{cm}^{2}\right)$ & 195 & 195 & 197 & 206 & 239 & 372 & 911 & 3087 & \\
\hline & Time & 260 & 154 & 91 & 54 & 32 & 19 & 12 & 8 & \\
\hline & AT product & 50679 & 30156 & 18006 & 11082 & 7643 & 7176 & 10884 & 24526 & \\
\hline \multirow{3}{*}{28} & Area $\left(\mathrm{cm}^{2}\right)$ & 232 & 233 & 235 & 243 & 276 & 411 & 956 & 3154 & 12029 \\
\hline & Time & 217 & 130 & 77 & 46 & 27 & 16 & 10 & 6 & 4 \\
\hline & AT product & 50380 & 30233 & 18102 & 11074 & 7445 & 6583 & 9214 & 18842 & 47789 \\
\hline \multirow{3}{*}{30} & Area $\left(\mathrm{cm}^{2}\right)$ & 386 & 386 & 388 & 397 & 431 & 567 & 1117 & 3338 & 12303 \\
\hline & Tim & 179 & 109 & 65 & 39 & 23 & 13 & 8 & 5 & 3 \\
\hline & AT product & 68947 & 41930 & 25241 & 15304 & 9813 & 7635 & 8940 & 16090 & 36749 \\
\hline
\end{tabular}

1. use 582 pieces of $300 \mathrm{~mm}$ silicon wafer.

2. operating frequency is $500[\mathrm{MHz}]$.

Totally, the total processing time Time ${ }^{(1024)}(k, m)$ is formulated as follows:

$$
\begin{aligned}
\text { Time }^{(1024)}(k, m) & =\frac{T_{\text {rout }}}{500[\mathrm{MHz}]} \cdot \frac{2 H_{a} \times H_{b}}{2^{k}} \cdot \frac{3}{4} \cdot \frac{1}{582} \cdot \frac{1}{365 \cdot 24 \cdot 3600} \\
& =\frac{2912.72\left(6.24-2 \log \log 2^{k-2 m}\right)}{2^{m}} \quad \text { (years) }
\end{aligned}
$$

\subsection{Optimizations of Parameters}

In this section, we optimize parameters $k, m$ in the sense of Area-Time product. Table 6 shows concrete values of circuit area and processing time of YASD1024 . From these data, we obtain optimized parameters as $k=26$ and $m=10$. In this case, an LSI requires as large as about $411.17 \mathrm{~cm}^{2}$ and 16 years for the sieving.

\section{Concluding Remarks}

This paper estimates the circuit area and the processing time of YASD for 1024bit integers. In the optimized parameters, namely $k=28$ and $m=10$, YASD requires at least $411 \mathrm{~cm}^{2}$ and 16 years for the sieving. Since we did not consider wiring problem in detail in our estimation, YASD1024 will reuire much more area and time even if it is manufactured and proceeded. 


\section{Acknowledgments}

The authors thank Takeshi Shimoyama for his helpful suggestions. We also thank anonymous reviewers for their comments.

\section{References}

[Ber01] Daniel J. Bernstein, "Circuits for Integer Factorization: A Proposal", preprint, 2001. http://cr.yp.to/papers/nfscircuit.pdf

[RSA200] Friedrich Bahr, M. Böhm, Jens Franke, and Thorsten Kleinjung, "RSA200", May 2005. http://www. crypto-world.com/announcements/rsa200.txt

[FKPP+05] Jens Franke, Thorsten Kleinjung, Christof Paar, Jan Pelzl, Christine Priplata, and Colin Stahlke, "SHARK: A Realizable Special Hardware Sieving Device for Factoring 1024-bit Integers", CHES 2005. pp.119-130, LNCS 3659, 2005.

[GT04] Willi Geiselmann, and Rainer Steinwandt, "Yet Another Sieving Device", CTRSA 2004, pp.278-291, LNCS 2964, 2004.

[GT03] Willi Geiselmann, and Rainer Steinwandt , "A Dedicated Sieving Hardware", PKC 2003 , pp. 254-266, LNCS 2567, 2002.

[LSTT02] Arjen K. Lenstra, Adi Shamir, Jim Tomlinson, and Eran Tromer, "Analysis of Bernstein's Factorization Circuit", ASIACRYPT 2002, pp. 1-26, LNCS 2501, 2002.

[LTSK+03] Arjen K. Lenstra, Eran Tromer, Adi Shamir, Wil Kortsmit, Bruce Dodson, James Hughes, and Paul C. Leyland, "Factoring Estimates for a 1024-Bit RSA Modulus", ASIACRYPT 2003, pp.55-74, LNCS 2894, 2003.

[Sha99] Adi Shamir, "Factoring Large Numbers with the TWINKLE Device (extended abstract)", CHES 1999, LNCS 1717, pp.2-12, Springer-Verlag, 1999.

[ST03] Adi Shamir, and Eran Tromer, "Factoring Large Number with the TWIRL Device", CRYPTO 2003, pp. 1-26, LNCS 2729, 2003. 